\title{
Performance Analysis of mmWave Communications with Selection Combining over Fluctuating-Two Ray Fading Model
}

\author{
Hussien Al-Hmood, Member, IEEE, and H. S. Al-Raweshidy, Senior Member, IEEE
}

\begin{abstract}
In this letter, the performance of millimeter-wave (mmWave) communications with selection combining (SC) over non-identical fluctuating two-ray (FTR) fading channels is analyzed. Consequently, the exact expressions and asymptotic approximations at high signal-to-noise ratio (SNR) regime of the moment generating function (MGF) and probability density function (PDF) of the maximum of non-identical FTR variates are provided. To this effect, mathematically tractable expressions of the outage probability (OP), outage capacity (OC), average bit error probability (ABEP), and average channel capacity (ACC) are derived. The truncation of the infinite series of the cumulative distribution function (CDF) for a specific number of terms is also given. A comparison between the numerical and simulated results is performed to verify the validation of our analysis.
\end{abstract}

Index Terms-Selection combining, fluctuating two-ray, outage probability, outage capacity, average bit error probability.

\section{INTRODUCTION}

$\mathbf{M}$ ILLIMETER-wave (mmWave) communications have been considered as the basis of the fifth-generation (5G) of wireless systems [1]. Hence, several efforts have been dedicated to model the channel of mmWave communications. In [2], the Rician distribution was used for small-scale fading in both line-of-sight (LoS) and non-LoS (NLoS) mediums. However, the Rician fading does not provide an accurate representation for the random fluctuating of the received signal. Therefore, to obtain close results to practical measurements of $28 \mathrm{GHz}$ outdoor mmWave channels, the authors in [3] proposed the fluctuating two-ray (FTR) fading model.

Based on the above observations, the probability density function (PDF), cumulative distribution function (CDF), and moment generating function (MGF) of the signal-to-noise ratio (SNR) over FTR fading channels were derived in [4] and applied to the average bit error probability (ABEP). The secrecy performance of the physical layer over FTR fading conditions was investigated in [5]. The secrecy outage probability (SOP) in cognitive radio networks (CRNs) over FTR fading was derived in [6]. The effective capacity of FTR fading channel was given in [7]. The average channel capacity (ACC) over FTR fading was studied in [8]. In [9], the authors analyzed the performance of a mixed free-space optics/mmWave system over Gamma-Gamma/FTR fading channels. The wireless powered unmanned aerial vehicle (UAV) relay over FTR fading model was presented in [10].

Manuscript received January 00, 2021

Hussien Al-Hmood is with the Department of Electrical and Electronics Engineering, College of Engineering, University of Thi-Qar, Iraq, e-mail: Hussien.Al-Hmood@utq.edu.iq.

H. S. Al-Raweshidy is with the Department of Electronic and Computer Engineering, College of Engineering, Design and Physical Sciences, Brunel University London, UK, e-mail: Hamed.Al-Raweshidy@brunel.ac.uk.
Recently, several works have been dedicated to analyze the statistical properties of the mathematical operations on the FTR random variables (RVs). For example, the distributions of the products and ratio of the products of FTR variates with independent and non-identically distributed (i.n.i.d.) fading severity indices were introduced in [11] and [12], respectively. In [13], the authors derived the PDF and CDF of the sum of arbitrarily distributed FTR variates with applications to the OP and ABEP of maximal ratio combining (MRC) diversity reception. The asymptotic and non-asymptotic expressions of the $\mathrm{OP}$ and ABEP with non-identically distributed MRC receivers over FTR fading channels were given in [14]. The 5G relay system for high-speed trains over FTR fading conditions with MRC scheme was considered in [15]. In [16], the statistics of the sum of the products of i.n.i.d. FTR variates were utilized to study the performance of mmWave communications with reconfigurable intelligent surface (RIS).

Motivated by the performance of mmWave communications with selection combining (SC) scheme over i.n.i.d. FTR fading channels has not been yet investigated in the literature, our main contributions in this letter are summarized as follows.

- Deriving both the exact expression and asymptotic approximation at high SNR values of the PDF and MGF of the maximum of non-identical FTR variates.

- Capitalizing on the above, mathematically tractable expressions of the OP, outage capacity (OC), ABEP, and ACC are obtained. In contrast to [17] in which the performance of mmWave communications with identical dual-branch SC scheme was investigated, our analysis can be used for $N$ receivers over i.n.i.d. FTR fading channels.

- Truncating the infinite series of the CDF up to a certain number of terms that satisfies a specific accuracy.

\section{Fluctuating Two-RAy FAding Channels}

The CDF of the instantaneous SNR at $l$ th receiver, $\gamma_{l}$, over FTR fading channel is expressed as [4, eq. (7)]

$$
F_{\gamma_{l}}(x)=\frac{m_{l}^{m_{l}}}{\Gamma\left(m_{l}\right)} \sum_{j_{l}=0}^{\infty} \frac{K_{l}^{j_{l}} d_{j_{l}}}{\left[\Gamma\left(j_{l}+1\right)\right]^{2}} \gamma\left(j_{l}+1, \frac{x}{2 \sigma_{l}^{2}}\right) \text {. }
$$

where

$$
\begin{aligned}
d_{j_{l}}= & \sum_{k_{l}=0}^{j_{l}}\left(\begin{array}{c}
j_{l} \\
k_{l}
\end{array}\right) \sum_{i_{l}=0}^{k_{l}}\left(\begin{array}{c}
k_{l} \\
i_{l}
\end{array}\right) \frac{\Gamma\left(j_{l}+m_{l}+2 i_{l}-k_{l}\right)}{\left(m_{l}+K_{l}\right)^{j_{l}+m_{l}+2 i_{l}-k_{l}}}\left(\frac{\Delta_{l}}{2}\right)^{2 i_{l}} \\
& \times K_{l}^{2 i_{l}-k_{l}}(-1)^{2 i_{l}-k_{l}} R_{j_{l}+m_{l}}^{k_{l}-2 i_{l}}\left(\left[\frac{K_{l} \Delta_{l}}{m_{l}+K_{l}}\right]^{2}\right) .
\end{aligned}
$$

with $m_{l}$ is the fading severity index, $K_{l}$ is the average power ratio between the dominant component to the scattering 


$$
\mathcal{M}_{\gamma}(s)=\sum_{j_{1}=0}^{\infty} \cdots \sum_{j_{N}=0}^{\infty}\left(\prod_{l=1}^{N} \frac{\Theta_{j_{l}}}{j_{l}+1}\right) \frac{\Gamma(1+\Xi)}{s^{\Xi}} F_{A}^{(N)}\left(1+\Xi, j_{1}+1, \cdots, j_{N}+1 ; j_{1}+2, \cdots, j_{N}+2 ;-\frac{1}{2 \sigma_{l}^{2} s}, \cdots,-\frac{1}{2 \sigma_{N}^{2} s}\right)
$$

$$
f_{\gamma}(x)=\sum_{j_{1}=0}^{\infty} \cdots \sum_{j_{N}=0}^{\infty}\left(\prod_{l=1}^{N} \Theta_{j_{l}}\right) x^{\Xi-1} H_{1,1:[1,2]]_{l=1: N}}^{0,1:[1,]_{l=1: N}}\left[\frac{x}{2 \sigma_{l}^{2}}, \cdots, \frac{x}{2 \sigma_{N}^{2}} \mid \begin{array}{c}
-\Xi ;(1)_{l=1: N}\left|\begin{array}{c}
\left(-j_{l}, 1\right)_{l=1: N} \\
1-\Xi ;(1)_{l=1: N}
\end{array}\right|\left[(0,1) ;\left(-1-j_{l}, 1\right)\right]_{l=1: N}
\end{array}\right] .
$$

multipath, $\Delta_{l} \in[0,1]$ is the similarity of two dominant waves, $2 \sigma_{l}^{2}=\bar{\gamma}_{l} /\left(1+K_{l}\right)$ with $\bar{\gamma}_{l}$ denotes the average SNR at $l$ th receiver, $\Gamma($.$) is the gamma function, \gamma(.,$.$) is the lower$ incomplete gamma function [19, eq. (8.350.1)], and $R_{v}^{\mu}(z)$ is defined as [18, eq. (20)]

$$
R_{v}^{\mu}(z)= \begin{cases}\left(\frac{v-\mu}{2}\right)_{\mu}\left(\frac{v-\mu+1}{2}\right) \frac{z^{\mu}}{\mu !} & \\ \times_{2} F_{1}\left(\frac{v+\mu}{2}, \frac{v+\mu+1}{2} ; 1+\mu ; z\right), \quad \mu \in \mathbb{N}^{+} \\ \frac{{ }_{2} F_{1}\left(\frac{v-\mu}{2}, \frac{v-\mu+1}{2} ; 1-\mu ; z\right)}{\Gamma(1-\mu)}, & \text { Otherwise }\end{cases}
$$

where $(.)_{a}$ is the Pochhammer symbol [19, eq. (1.2.2)].

The asymptotic of the CDF in (1) at $\bar{\gamma}_{l} \rightarrow \infty$ for $l=$ $1, \cdots, N$, is given by [6, eq. (18)]

$$
F_{\gamma_{l}}^{\infty}(x) \approx \frac{m_{l}\left(1+K_{l}\right) d_{j_{l}=0}}{\Gamma\left(m_{l}\right) \bar{\gamma}_{l}} x
$$

where $d_{j_{l}=0}$ is the value of $d_{j_{l}}$ in (2) at $j_{l}=0$.

\section{Statistics of the Maximum of FTR Variates}

Proposition 1: Assume all RVs, $\gamma_{l} \sim$ $\mathcal{F} \mathcal{T} \mathcal{R}\left(m_{l}, K_{l}, \Delta_{l}, 2 \sigma_{l}^{2}\right)$ for $l \in\{1, \cdots, N\}$ where $N$ is the number of the variates, follow i.n.i.d. FTR distribution, the MGF of $\gamma=\max \left\{\gamma_{1}, \cdots, \gamma_{N}\right\}$ is provided in (5) shown at the top of this page, where $\Theta_{j_{l}} \triangleq \frac{m_{l}^{m_{l}} K_{l}^{j_{l}} d_{j_{l}}}{\Gamma\left(m_{l}\right)\left(2 \sigma_{l}^{2}\right)^{j_{l}+1} \Gamma\left(j_{l}+1\right) j_{l} !}$ and $\Xi \triangleq N+\sum_{l=1}^{N} j_{l}$.

It can be observed that (5) is written in terms of the multivariate Lauricella hypergeometric function, $F_{A}^{(N)}(),.[20$, eq. (1.7.1)]. Although this function is not yet implemented in the popular mathematical software packages, it can be efficiently computed via downloading a MATLAB code from [21].

The asymptotic approximation of the MGF at high average SNR regime is obtained as

$$
\mathcal{M}_{\gamma}^{\infty}(s) \approx\left(\prod_{l=1}^{N} \frac{m_{l}\left(1+K_{l}\right) d_{j_{l}=0}}{\Gamma\left(m_{l}\right) \bar{\gamma}_{l}}\right) \frac{\Gamma(1+N)}{s^{N}} .
$$

Proof: The CDF of the maximum i.n.i.d. variates can be evaluated as

$$
F_{\gamma}(x)=\prod_{l=1}^{N} F_{\gamma_{l}}(x)
$$

Substituting (1) into (7) and invoking the identity [22, eq. (6.5.12)], this yields

$$
\begin{aligned}
& F_{\gamma}(x)=\sum_{j_{1}=0}^{\infty} \cdots \sum_{j_{N}=0}^{\infty}\left(\prod_{l=1}^{N} \frac{\Theta_{j_{l}}}{j_{l}+1}\right) \\
& x^{\Xi} \prod_{l=1}^{N}{ }_{1} F_{1}\left(j_{l}+1 ; j_{l}+2 ;-\frac{x}{2 \sigma_{l}^{2}}\right) .
\end{aligned}
$$

Plugging (8) in $\mathcal{M}_{\gamma}(s)=s \mathcal{L}\left[F_{\gamma}(x) ; s\right]$ where $\mathcal{L}[$.$] indicates$ the Laplace transform, and recalling [20, eq. (2.i), p. 260], the proof of (5) is accomplished.

Inserting (4) in (7), the asymptotic of the CDF of the maximum i.n.i.d. FTR variates is obtained as

$$
F_{\gamma}^{\infty}(x) \approx\left(\prod_{l=1}^{N} \frac{m_{l}\left(1+K_{l}\right) d_{j_{l}=0}}{\Gamma\left(m_{l}\right) \bar{\gamma}_{l}}\right) x^{N} .
$$

Next, inserting (9) in $\mathcal{M}_{\gamma}^{\infty}(s)=s \mathcal{L}\left[F_{\gamma}^{\infty}(x) ; s\right]$ and making use of [19, eq. (3.381.4)], (6) is deduced and this finishes the proof.

Proposition 2: The PDF of $\gamma$ is given in (10) presented at the top of this page and the asymptotic expression of the PDF is derived as

$$
f_{\gamma}^{\infty}(x) \approx N\left(\prod_{l=1}^{N} \frac{m_{l}\left(1+K_{l}\right) d_{j_{l}=0}}{\Gamma\left(m_{l}\right) \bar{\gamma}_{l}}\right) x^{N-1} .
$$

In (10), $H_{1,1:[c, d]_{l=1: N}^{0,1}[.]}$ denotes the multivariate $H$ function defined in [23, eq. (A.1)]. This function is not available as a built-in function in MATLAB and MATHEMATICA software packages. Thus, a MATLAB code that is written in [24] can be used to compute this function.

Proof: With the help of [25, eq. (1.10.7)], the MGF of $\gamma$ that is given in (5) can be expressed in multiple Barnes-type closed integral contours as

$$
\begin{gathered}
\mathcal{M}_{\gamma}(s)=\sum_{j_{1}=0}^{\infty} \cdots \sum_{j_{N}=0}^{\infty} \frac{\left(\prod_{l=1}^{N} \Theta_{j_{l}}\right)}{s^{\Xi}} \frac{1}{(2 \pi i)^{N}} \int_{\mathcal{T}_{1}} \cdots \int_{\mathcal{T}_{N}} \\
\Gamma\left(1+\Xi+\sum_{l=1}^{N} t_{l}\right)\left\{\prod_{l=1}^{N} \frac{\Gamma\left(1+j_{l}+t_{l}\right) \Gamma\left(-t_{l}\right)}{\Gamma\left(2+j_{l}+t_{l}\right)}\left(\frac{1}{2 \sigma_{l}^{2} s}\right)^{t_{l}}\right\} \\
d t_{1} \cdots d t_{N} .
\end{gathered}
$$

where $\mathcal{T}_{l}$ is the $l$ th suitable contour in the $t$-plane from $\nu_{l}-i \infty$ to $\nu_{l}+i \infty$ with $\nu_{l}$ is a constant number.

Plugging (12) in $f_{\gamma}(x)=\mathcal{L}^{-1}\left[\mathcal{M}_{\gamma}(s) ; x\right]$ where $\mathcal{L}^{-1}[$. represents the inverse Laplace transform and applying the Fubini's theorem to interchange the order of the integrations, 


$$
P_{b}=\sum_{j_{1}=0}^{\infty} \cdots \sum_{j_{N}=0}^{\infty}\left(\prod_{l=1}^{N} \frac{\Theta_{j_{l}}}{j_{l}+1}\right) \frac{\left(\rho_{2}\right) \Xi}{2 \rho_{1}^{\Xi}} F_{A}^{(N)}\left(\rho_{2}+\Xi, j_{1}+1, \cdots, j_{M}+1 ; j_{1}+2, \cdots, j_{M}+2 ;-\frac{1}{2 \sigma_{l}^{2} \rho_{1}}, \cdots,-\frac{1}{2 \sigma_{M}^{2} \rho_{1}}\right) .
$$

this deduces

$$
\begin{gathered}
f_{\gamma}(x)=\sum_{j_{1}=0}^{\infty} \cdots \sum_{j_{N}=0}^{\infty}\left(\prod_{l=1}^{N} \Theta_{j_{l}}\right) \frac{1}{(2 \pi i)^{N}} \int_{\mathcal{T}_{1}} \cdots \int_{\mathcal{T}_{N}} \\
\Gamma\left(1+\Xi+\sum_{l=1}^{N} t_{l}\right)\left\{\prod_{l=1}^{N} \frac{\Gamma\left(1+j_{l}+t_{l}\right) \Gamma\left(-t_{l}\right)}{\Gamma\left(2+j_{l}+t_{l}\right)}\left(\frac{1}{2 \sigma_{l}^{2}}\right)^{t_{l}}\right\} \\
\mathcal{L}^{-1}\left[s^{-\Xi-\sum_{l=1}^{N} t_{l}} ; x\right] d t_{1} \cdots d t_{N} .
\end{gathered}
$$

Evaluating the inverse Laplace transform of (13) via recalling [19, eq. (17.13.3)] and then employing [23, eq. (A.1)], the result is (10).

The asymptotic of the PDF can be easily derived via utilizing $f_{\gamma}^{\infty}(x)=d F_{\gamma}^{\infty}(x) / d x$. Furthermore, (11) can be obtained via substituting (6) into $f_{\gamma}^{\infty}(x)=\mathcal{L}^{-1}\left[\mathcal{M}_{\gamma}^{\infty}(s) ; x\right]$ and invoking [19, eq. (17.13.3)/ eq. (8.331.1)] which completes the proof.

\section{Performance Analysis of SC Scheme}

Due to its low implementation complexity, the SC diversity reception has been widely used to improve the performance of wireless communication systems. In SC, the receiver with the highest SNR is selected among number of branches.

\section{A. Outage Probability}

The OP is employed to measure the performance of wireless communication systems over fading channels. It can be defined as the probability of the instantaneous SNR at the output of the combiner falls below the predetermined threshold $\lambda$, namely, $P_{o}=\operatorname{Pr}\{\gamma<\lambda\}$ where $\operatorname{Pr}\{$.$\} denotes the probability.$

The OP can be evaluated by [9, eq. (25)]

$$
P_{o}=F_{\gamma}(\lambda) \text {. }
$$

To this effect, the OP and its asymptotic behaviour of mmWave communications with SC scheme can be readily obtained by using (8) and (9), respectively.

It is worth noting that the asymptotic of the OP can be further approximated as $P_{o}^{\infty} \approx \bar{\gamma}^{-G_{d}}$ where $G_{d}$ is the diversity order. From (9), one can see that $G_{d}$ is proportional to the number of branches, $N$. This result is matched with [13] that is given for MRC diversity.

\section{B. Outage Capacity}

The OC is used to quantify the spectral efficiency over fading channels. It can be defined as the probability of the instantaneous capacity $C_{\gamma}$ is less than a certain threshold value $\varphi$, that is, $C_{o}=\operatorname{Pr}\left\{0 \leqslant C_{\gamma}<\varphi\right\}$, where $C_{\gamma}=B \log _{2}(1+\gamma)$ and $B$ is the bandwidth of the transmitted signal. Consequently, the $\mathrm{OC}$ can be expressed as

$$
C_{o}=F_{\gamma}\left(2^{\varphi / B}-1\right) \text {. }
$$

Based on (15), the exact and asymptotic expressions of the OC of mmWave communications with $\mathrm{SC}$ receivers can be evaluated by (8) and (9), respectively.

\section{Average Bit Error Probability}

The ABEP can be calculated by [4, eq. (15)]

$$
P_{b}=\frac{\rho_{1}^{\rho_{2}}}{2 \Gamma\left(\rho_{2}\right)} \int_{0}^{\infty} x^{\rho_{2}-1} e^{-\rho_{1} x} F_{\gamma}(x) d x .
$$

where $\left(\rho_{1}, \rho_{2}\right)=(1,0.5)$ for binary phase shift keying (BPSK) and $\left(\rho_{1}, \rho_{2}\right)=(0.5,0.5)$ for coherent binary frequency shift keying (C-BFSK) modulations.

Inserting (8) in (16) and making utilize of [20, eq. (2.i), p. 260], the ABEP is deduced as in (17) given at the top of this page.

When $\bar{\gamma}_{l} \rightarrow \infty$ for $l=1, \cdots, N$, the asymptotic of the ABEP is derived via substituting (9) into (16) and recalling [19, eq. (3.381.4)]. Thus, this yields

$$
P_{b}^{\infty} \approx\left(\prod_{l=1}^{N} \frac{m_{l}\left(1+K_{l}\right) d_{j_{l}=0}}{\Gamma\left(m_{l}\right) \bar{\gamma}_{l}}\right) \frac{\left(\rho_{2}\right)_{N}}{2 \rho_{1}^{N}} .
$$

It is evident that $G_{d}$ of the ABEP also depends on $N$.

\section{Average Channel Capacity}

The normalized ACC can be computed by [4, eq. (11)]

$$
C=\frac{1}{\ln (2)} \int_{0}^{\infty} \ln (1+x) f_{\gamma}(x) d x
$$

Plugging (10) in (19) and using [23, eq. (A.1)], this yields

$$
\begin{aligned}
& C=\frac{1}{\ln (2)} \sum_{j_{1}=0}^{\infty} \cdots \sum_{j_{N}=0}^{\infty}\left(\prod_{l=1}^{N} \Theta_{j_{l}}\right) \frac{1}{(2 \pi i)^{N}} \int_{\mathcal{T}_{1}} \cdots \int_{\mathcal{T}_{N}} \\
& \frac{\Gamma\left(1+\Xi+\sum_{l=1}^{N} t_{l}\right)}{\Gamma\left(\Xi+\sum_{l=1}^{N} t_{l}\right)}\left\{\prod_{l=1}^{N} \frac{\Gamma\left(1+j_{l}+t_{l}\right) \Gamma\left(-t_{l}\right)}{\Gamma\left(2+j_{l}+t_{l}\right)}\left(\frac{1}{2 \sigma_{l}^{2}}\right)^{t_{l}}\right\} \\
& \int_{0}^{\infty} x^{\Xi+\sum_{l=1}^{N} t_{l}-1} \ln (1+x) d x d t_{1} \cdots d t_{N} .
\end{aligned}
$$

The inner integration of (20) is recorded in [19, eq. (4.293.3)]. Thus, after recalling the identity [20, eq. (1.1.8)] and doing some mathematical simplifications, we have

$$
\begin{aligned}
& C=\frac{1}{\ln (2)} \sum_{j_{1}=0}^{\infty} \cdots \sum_{j_{N}=0}^{\infty}\left(\prod_{l=1}^{N} \Theta_{j_{l}}\right) \frac{1}{(2 \pi i)^{N}} \int_{\mathcal{T}_{1}} \cdots \int_{\mathcal{T}_{N}} \\
& \Gamma\left(\Xi+\sum_{l=1}^{N} t_{l}\right) \Gamma\left(1-\Xi-\sum_{l=1}^{N} t_{l}\right) \\
& \left\{\prod_{l=1}^{N} \frac{\Gamma\left(1+j_{l}+t_{l}\right) \Gamma\left(-t_{l}\right)}{\Gamma\left(2+j_{l}+t_{l}\right)}\left(\frac{1}{2 \sigma_{l}^{2}}\right)^{t_{l}}\right\} d t_{1} \cdots d t_{N} .
\end{aligned}
$$




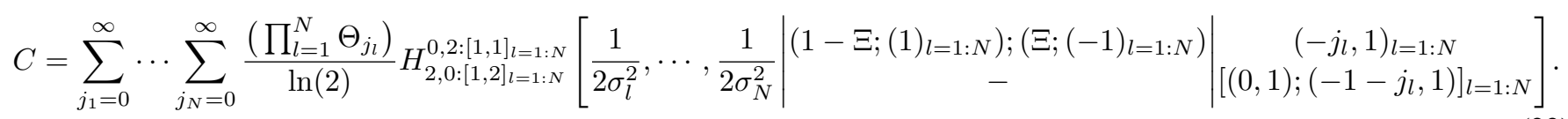

TABLE I

REQUIRED TERMS $M_{l}$ FOR THE TRUNCATION ERROR $\left(\epsilon\left(M_{l}\right) \leq 10^{-7}\right)$ FOR DIFFERENT $N$ AND FADING PARAMETERS.

\begin{tabular}{|c|c|c|c|c|c|}
\hline$N$ & $m_{l}$ & $K_{l}$ & $\Delta_{l}$ & $M_{l}$ & $\epsilon$ \\
\hline \hline 1 & 0.5 & 0.5 & 0.3 & 36 & $5.0461 \times 10^{-8}$ \\
\hline 2 & 0.5 & 0.5 & 0.3 & 38 & $5.3024 \times 10^{-8}$ \\
\hline 2 & 1.5 & 1.5 & 0.5 & 33 & $7.1688 \times 10^{-8}$ \\
\hline 3 & 1.5 & 2.5 & 0.5 & 25 & $9.5214 \times 10^{-8}$ \\
\hline 4 & 1.5 & 2.5 & 0.5 & 19 & $3.7726 \times 10^{-8}$ \\
\hline
\end{tabular}

With the aid of [23, eq. (A.1)], the exact expression of $C$ is yielded as in (22) provided at the top of this page.

The asymptotic expression of the normalized ACC at $\bar{\gamma}_{l} \rightarrow$ $\infty$, can be calculated by [26, eq. (27)]

$$
C^{\infty} \approx \frac{1}{\ln (2)} \sum_{r=0}^{T} w_{r} \frac{1-\mathcal{M}_{\gamma}^{\infty}\left(y_{r}\right)}{y_{r}} .
$$

where $\mathcal{M}_{\gamma}^{\infty}($.$) is given in (6), T$ is the number of terms for the Gaussian-Laguerre integration whereas $w_{r}$ and $y_{1}$ are the weight and abscissas factors, respectively [22].

\section{Truncating Error of the Derived Statistics}

It is apparent that all the derived expressions include multiple infinite series. Therefore, the convergence acceleration with a certain figure of accuracy should be applied with truncating error $\epsilon\left(M_{l}\right)$ that is given by [13, eq. (10)]

$$
\epsilon\left(M_{l}\right) \triangleq F_{\gamma}(\infty)-\hat{F}_{\gamma}(\infty) .
$$

where $\hat{F}_{\gamma}(\infty)$ is the truncated CDF up to $M_{l}$ first terms.

Using the fact that $\lim _{x \rightarrow \infty} F_{\gamma}(x)=1$ and substituting (1) into (7) along with the relation $\lim _{x \rightarrow \infty} \gamma\left(j_{l}+1, \frac{x}{2 \sigma_{l}^{2}}\right)=1$, (24) becomes

$$
\epsilon\left(M_{l}\right)=1-\sum_{j_{1}=0}^{M_{1}} \cdots \sum_{j_{N}=0}^{M_{N}} \prod_{l=1}^{N} \frac{m_{l}^{m_{l}} K_{l}^{j_{l}} d_{j_{l}}}{\Gamma\left(m_{l}\right) \Gamma\left(j_{l}+1\right) j_{l} !} .
$$

From (25), it is clear that $\epsilon\left(M_{l}\right) \rightarrow 0$ as $M_{l} \rightarrow \infty$ for $l=1, \cdots, N$.

Table I explains the required truncation terms $M_{l}$ for different $N$ and fading parameters of the channel. It can be noted that 40 terms are enough for all diversity branches to obtain highly accurate results (e.g., $\epsilon\left(M_{l}\right) \leq 10^{-7}$ ) in all considered cases.

\section{Analytical and Simulation Results}

In this section, the exact analytical and simulated results are presented for different scenarios of mmWave communications with SC diversity reception over FTR fading channels.

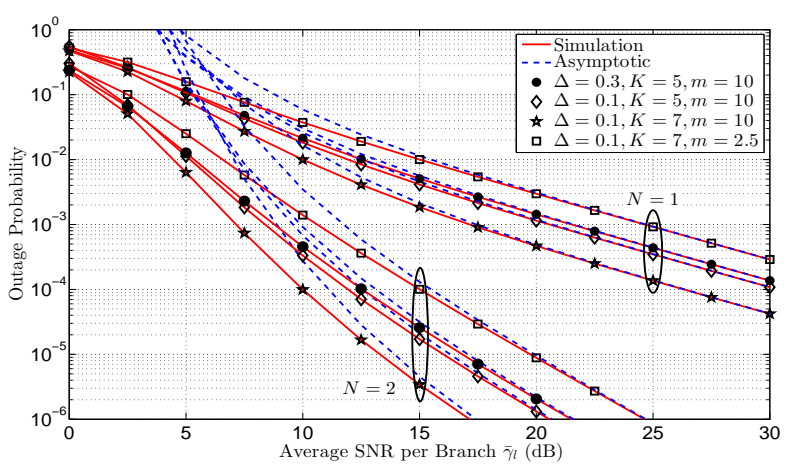

Fig. 1. OP versus $\bar{\gamma}_{l}$ for different $\Delta, K, m, N=1, N=2$ and $\lambda=0 \mathrm{~dB}$.

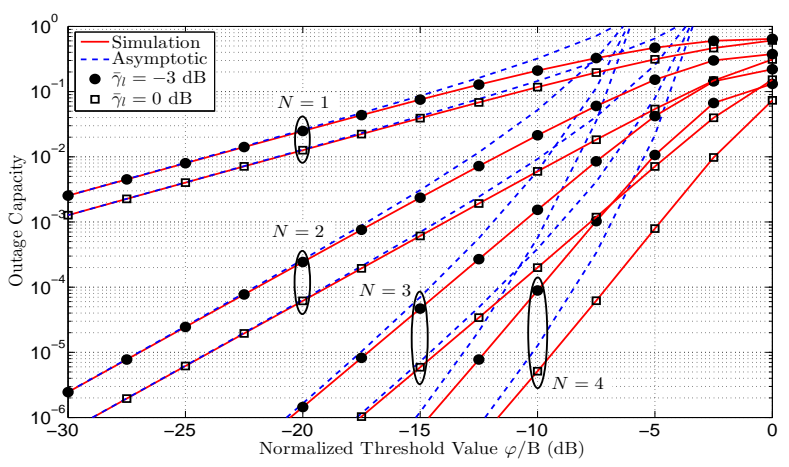

Fig. 2. OC versus normalized threshold value for different $N$ and $\bar{\gamma}_{l}$.

Fig. 1 depicts the OP versus $\bar{\gamma}_{l}$ for different values of the fading parameters and $\lambda=0 \mathrm{~dB}$ of mmWave communications with single receiver and dual-branch SC scheme over identically distributed FTR fading channels. From this figure, it is clear that the OP is remarkably diminished when the SC scheme is employed. This is due to the increasing in the diversity gain which is related to $N$. Furthermore, the OP decreases as $m$ or/and $K$ increase. This is because the increasing in $m$ corresponds to less fluctuating in the received signal whereas a high value of $K$ means the total power of the dominant components is larger than the total power of the scattered waves. On contrary, the OP sightly degrades as $\Delta$ increases. The reason is that a higher value of $\Delta$ corresponds to a large phase difference between the two dominant waves. These observations are consistent with the results in [4] and [13] that are given for no diversity and MRC scheme, respectively.

Fig. 2 plots the OC versus $\varphi / B$ for $\bar{\gamma}=0$ and $-3 \mathrm{~dB}$ of mmWave communications with single branch and dual, triple, and quadruple SC receivers over non-identical FTR fading channels whilst Fig. 3 demonstrates the ABEP for BPSK and C-BFSK modulations versus $\bar{\gamma}_{l}$. In these figures, the fading parameters are $m_{1}=0.5, m_{2}=1.5, m_{3}=2.5, m_{4}=3.5$, 


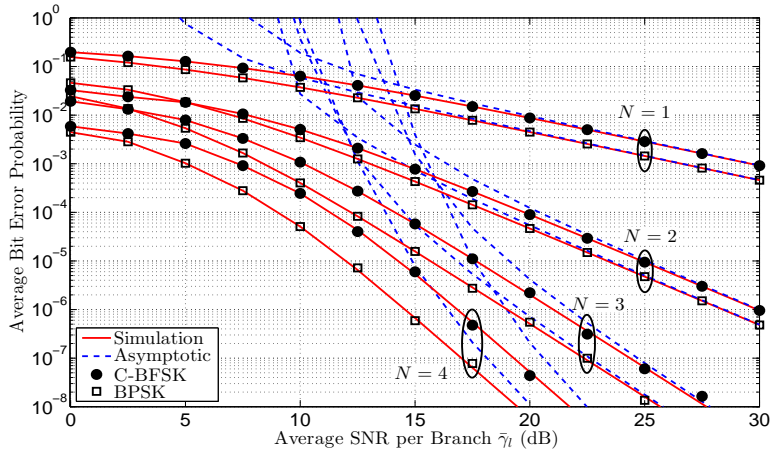

Fig. 3. ABEP versus average SNR for different $N$ and modulation schemes.

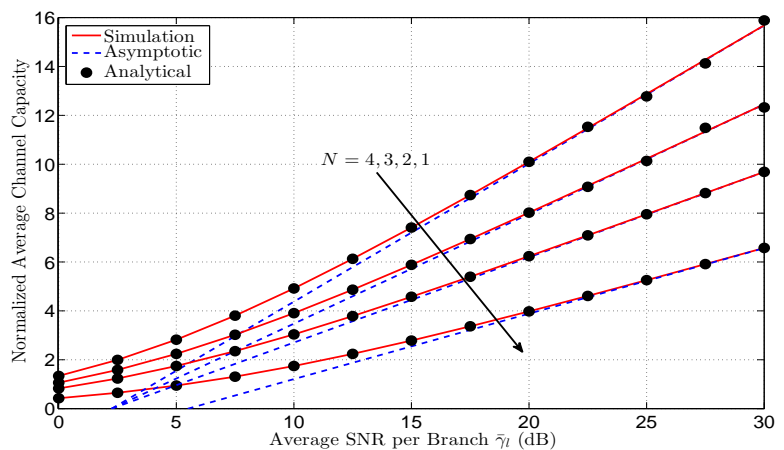

Fig. 4. ACC versus average SNR for different $N$.

$K_{l}=5$, and $\Delta_{l}=0.3$ for $l=1,2,3,4$. In both figures, as anticipated, the performance of the considered system can be further improved via increasing the number of the diversity receivers. This is because a larger branch number can achieve higher diversity gain. For example, in Fig. 3, when $\bar{\gamma}_{l}$ is constant at $10 \mathrm{~dB}$, the ABEP of the BPSK modulation for $L=3$ is roughly $88.2 \%$ and $98.9 \%$ lower than the ABEP of $L=1$ and $L=2$, respectively. In the same context, the BPSK modulation outperforms the C-BFSK modulation, which is in line with the results in the open literature. Additionally, the results of Figs 2 and 3 are confirmed in Fig. 4 which explains the normalized ACC versus $\bar{\gamma}_{l}$. In this figure, in order to obtain highly accurate results for the asymptotic of the ACC, we have used $T=15$.

In all figures, an excellent agreement between the numerical and simulated results can be noticed, which proves the correctness of our derived expressions. Moreover, these results are matched with the asymptotic behaviour at $\bar{\gamma}_{l}$.

\section{CONCLUSIONS}

In this letter, the performance of mmWave communications with SC receivers over non-identical FTR fading channels was studied. To this effect, both the exact and asymptotic expressions of the CDF, MGF and PDF of the maximum i.n.i.d. variates were provided first. Thereafter, the OP, OC, ABEP, ACC were derived in mathematically tractable exact expressions. The results showed that the system performance can be greatly enhanced via using the SC diversity reception.

\section{REFERENCES}

[1] T. S. Rappaport, S. Sun, R. Mayzus, H. Zhao, Y. Azar, K. Wang, G. N. Wong, J. K. Schulz, M. Samimi, and F. Gutierrez, "Millimeter wave mobile communications for $5 \mathrm{G}$ cellular: It will work!," IEEE Access, vol. 1, pp. 335-349, May 2013.

[2] M. K. Samimi et al., " $28 \mathrm{GHz}$ millimeter-wave ultrawideband small-scale fading models in wireless channels," in Proc. IEEE 83rd Veh. Technol. Conf. (VTC Spring), May 2016, pp. 1-6.

[3] J. M. Romero-Jerez et al., "The fluctuating two-ray fading model: Statistical characterization and performance analysis," IEEE Trans. Wireless Commun., vol. 16, no. 7, pp. 4420-4432, Jul. 2017.

[4] J. Zhang et al., "New results on the fluctuating two-ray model with arbitrary fading parameters and its applications," IEEE Trans. Veh. Technol., vol. 67, no. 3, pp. 2766-2770, Mar. 2018.

[5] W. Zeng, J. Zhang, S. Chen, K. P. Peppas, and B. Ai, "Physical layer security over fluctuating two-ray fading channels," IEEE Trans. Veh. Technol., vol. 67, no. 9, pp. 8949-8953, Sep. 2018.

[6] H. Zhao, J. Zhang, L. Yang, G. Pan, and M.-S. Alouini, "Secure mmWave communications in cognitive radio networks," IEEE Wireless Commun. Lett., vol. 8, no. 4, pp. 1171-1174, Aug. 2019.

[7] K. P. Peppas et al., "Effective capacity of fluctuating two-ray channels with arbitrary fading parameters," in in IEEE SPAWC, 2018, pp. 1-5.

[8] H. Zhao, Z. Liu, and M.-S. Alouini, "Different power adaption methods on fluctuating two-ray fading channels," IEEE Wireless Commun. Lett., vol. 8, no. 2, pp. 592-595, Apr. 2019.

[9] Y. Zhang, J. Zhang, L. Yang,B. Ai, and M.-S. Alouini, "On the performance of dual-hop systems over mixed FSO/mmWave fading channels," IEEE Open J. Commun. Society, vol. 1, 2020, pp. 477-489, May 2020.

[10] J. Zheng et al., "Wireless powered UAV relay communications over fluctuating two-ray fading channels," Phys. Commun., vol. 35, May 2019.

[11] O. S. Badarneh and D. B. da Costa, "Cascaded fluctuating two-ray fading channels," IEEE Commun. Lett., vol. 23, no. 9, pp. 1497-1500, Sep. 2019.

[12] O. S. Badarneh et al., "Ratio of products of fluctuating two-ray variates," IEEE Commun. Lett., vol. 23, no. 11, pp. 1944-1948, Nov. 2019.

[13] J. Zheng, J. Zhang, G. Pan, J. Cheng, and B. Ai, "Sum of squared fluctuating two-ray random variables with wireless applications," IEEE Trans. Veh. Technol., vol. 68, no. 8, pp. 8173-8177, Aug. 2019.

[14] H. Hashemi, J. Haghighat, M. Eslami, and K. Navaie, "Amplify-andforward relaying with maximal ratio combining over fluctuating two-ray channel: Non-asymptotic and asymptotic performance analysis," IEEE Trans. Commun., vol. 68, no. 12, pp. 7446-7459, Dec. 2020.

[15] J. Zhang et al., "Performance analysis of $5 \mathrm{G}$ mobile relay systems for high-speed trains," IEEE J. Sel. A. Commun., vol. 38, no. 12, Dec. 2020.

[16] H. Du, J. Zhang, J. Cheng, and B. Ai, "Millimeter wave communications with reconfigurable intelligent surfaces: Performance analysis and optimization," IEEE Trans. Veh. Technol., vol. 69, no. 4, 2752-2768, Jan. 2021.

[17] L. M. Devi and A. D. Singh, "Performance of dual-branch selection combining receiver over fluctuating two-ray fading channels for 5G mmwave communications," Int. J. Electron. Commun. (AEU), vol. 117, Apr. 2020.

[18] M. López-Benítez, and J. Zhang, "Comments and corrections to "New results on the fluctuating two-ray model with arbitrary fading parameters and its applications," "IEEE Trans. Veh. Technol., vol. 70, no. 2, pp. 1938-1940, Feb. 2021.

[19] I. S. Gradshteyn, and I. M. Ryzhik, Table of Integrals, Series and Products, 7th edition. Academic Press Inc., 2007.

[20] H. M. Srivastava and H. L. Manocha, A Treatise on Generating Functions. New York, NY, USA: Wiley, 1984.

[21] Code for Calculating $F_{A}^{(N)}($.$) . [Online]. Available:$ http://faculty.smu.edu/rbutler/

[22] M. Abramowitz and I. A. Stegun, Handbook of Mathematical Functions: With Formulas, Graphs, and Mathematical Tables. New York, NY, USA: Dover, 1965.

[23] A. M. Mathai, R. K. Saxena, and H. J. Haubold, The H-Function: Theory and Applications, 1st edition. New York: Springer, 2010.

[24] H. Chergui et al., "Rician $K$-factor-based analysis of XLOS service probability in $5 \mathrm{G}$ outdoor ultra-dense networks," IEEE Wireless Commun. Lett., vol. 8, no. 2, pp. 428-431, Apr. 2018.

[25] A. M. Mathai and H. J. Haubold, Special functions for applied scientists, 1st edition. New York: Springer, 2008.

[26] H. Al-Hmood and H. S. Al-Raweshidy, "On the sum and the maximum of non-identically distributed composite $\eta$ - $\mu$ /gamma variates using a mixture gamma distribution with applications to diversity receivers," IEEE Trans. Veh. Technol., vol. 65, no. 12, pp. 10048-10052, Dec. 2016. 